\title{
ANALYSIS OF BOOST VOLUME DEFINITION IN RADIATION THERAPY FOR CONSERVATIVE BREAST SURGERY
}

Thiago Brasileiro de Freitas', Kennya Medeiros Lopes de Barros Lima', Heloísa de Andrade Carvalho², Alexandre Siqueira Franco Fonseca ${ }^{3}$, Alexandre Mendonça Munhoz ${ }^{3,4}$, José Roberto Filassi ${ }^{5}$, Silvia R. Stuart², Gustavo Nader Marta ${ }^{6 *}$

'Department of Radiology and Oncology, Division of Radiation Oncology, Faculdade de Medicina da Universidade de São Paulo - São Paulo (SP), Brazil.

2Department of Radiology and Oncology, Division of Radiation Oncology, Instituto de Radiologia (INRAD), Faculdade de Medicina da Universidade de São Paulo. Department of Radiation Oncology, Hospital Sírio-Libanês - São Paulo (SP), Brazil. ${ }^{3}$ Department of Plastic Surgery - Division of Breast Reconstruction, Cancer Institute of São Paulo, Faculdade de Medicina da Universidade de São Paulo - São Paulo (SP), Brazil.

${ }^{4}$ Division of Plastic Surgery, Hospital Sírio-Libanês - São Paulo (SP), Brazil.

${ }^{5}$ Department of Obstetrics and Gynecology, Faculdade de Medicina da Universidade de São Paulo - São Paulo (SP), Brazil. ${ }^{6}$ Department of Radiology and Oncology, Division of Radiation Oncology, Instituto do Câncer do Estado de São Paulo (ICESP), Faculdade de Medicina da Universidade de São Paulo. Department of Radiation Ongology, Hospital Sírio-Libanês São Paulo (SP), Brazil.

*Corresponding author: gustavo.marta@hc.fm.usp.br

Purpose/Objective(s): The surgical bed clipping in breast conserving surgery is not a worldwide systematic practice, leading to a major difficulty in the definition of the boost volume. In practice, when the surgical bed is not marked, to compensate for uncertainties, the boost dose is given to the whole quadrant (tumor pre-surgical clinical location). The purpose of this study was to evaluate the role of surgical clips placement in the definition of boost treatment volume. Materials/Methods: Clinical Target Volumes (CTV) were defined as: CTV Breast, CTV Quadrant (based on physical exam and pre-surgical images), CTV Boost, defined by clip plus margin ( $1 \mathrm{~cm}$ for 2 or more clips and $2 \mathrm{~cm}$ for 1 clip only) plus radiological changes, CTV NT (normal tissue), defined by CTV Quadrant minus CTV Boost and CTV MISS (CTV that would be outside the treatment volume), defined by CTV Boost minus CTV Quadrant. Results: A total of 247 patients were included. Upper lateral quadrant was the most common clinical location (47.3\%). The median number of clips used was three. The mean volumes were: CTV Breast: 982.52cc, CTV Boost: 36.59cc, CTV Quadrant: 285.07cc, CTV NT: 210.1cc and CTV MISS: 13.57cc. Only 50.6\% (125) of the patients presented the CTV Boost completely inside the CTV Quadrant and in 47.3\% (117), partially inside. Among patients with any CTV MISS, 80.3\% (98) had 10\% or more of CTV Boost outside the treatment volume. Regarding CTV MISS, there were no statistically significant differences between the groups with 1 clip versus 2 or more clips, nor between patients with or without reconstructive surgery. In average, the CTV Boost was $87 \%$ smaller than the CTV Quadrant. The whole quadrant irradiation would lead to unnecessary irradiation of $26 \%$ of normal breast tissue. Conclusion: Surgical bed clipping is up most important in the definition of the boost volume irradiation to ensure precision minimizing geographical miss and optimizing surrounding normal tissue sparing. 